\title{
Characteristic Morphological Changes and Rapid Actin Accumulation in Serum-MAF-treated Macrophages
}

\author{
KUMPEI KAWAKATSU ${ }^{1}$, MAMI ISHIKAWA ${ }^{1,2}$, RIHO MASHIBA ${ }^{1}$, \\ NGOC KIET TRAN ${ }^{1}$, MINORI AKAMATSU ${ }^{1}$ and TAKAHITO NISHIKATA ${ }^{1}$ \\ ${ }^{1}$ Frontiers of Innovative Research in Science and Technology (FIRST), Konan University, Kobe, Japan; \\ ${ }^{2}$ Aeras Bio Inc., Kobe, Japan
}

\begin{abstract}
Background/Aim: Serum-derived macrophage activating factor, serum-MAF, is known to increase the phagocytic activity of macrophages by enhancing the engulfment efficiency. To elucidate the mechanisms underlying phagocytic activation, morphological changes were observed and analyzed. Materials and Methods: Morphological changes in macrophages were observed and quantitatively analyzed using scanning electron microscope (SEM) and confocal microscope. Results: SEM and confocal microscopy images revealed frill-like structures and active actin accumulations, respectively, in serum-MAF treated macrophages. Actin accumulation was induced within 5 min following serum-MAF treatment. Conclusion: Serum-MAF induced a rapid rearrangement of cytoskeletal actin and enhanced phagocytic activity. Findings of the current study may contribute to the development of techniques that facilitate activation of the human immune system, which in turn may be beneficial for cancer immunotherapy.
\end{abstract}

The immune system consists of innate and adaptive components, each of which has its own function and role (1). Macrophages play an important role in the innate immune system by phagocytosing invading pathogens. Moreover, they play a crucial role by secreting cytokines and processing pathogen related information (1-3). Thus, enhanced activation of macrophages may activate the entire immune system.

Correspondence to: Takahito Nishikata, Frontiers of Innovative Research in Science and Technology (FIRST), Konan University, 7-1-20, Minatojima-minamimachi, Chuo-ku, Kobe 605-0047, Japan. Tel: +81 783031349, Fax: +81 783031495, e-mail: nisikata@konanu.ac.jp

Key Words: THP-1, serum-MAF (serum-derived macrophage activating factor), morphological change, actin accumulation, timelapse image.
Previously, we have established a novel experimental system for quantitatively evaluating the phagocytic activity of a human monocytic cell line derived from an acute monocytic leukemia patient (THP-1) derived macrophages (4). This system enables the evaluation of macrophage phagocytic activity within $60 \mathrm{~min}$ of macrophage activating factor (MAF) treatment. Among the well-known MAFs, serum-derived macrophage activating factor (serum-MAF), was found to elicit strong phagocytic activation $(4,5)$. This activation was achieved by enhancement of engulfment efficiency and not by activation of cell motility (6). The objective of the current study was to investigate the mechanisms underlying phagocytic activation and examine morphological changes in serum-MAF-activated macrophages.

\section{Materials and Methods}

Cell culture. The THP-1 cell line was obtained from RIKEN BRC (Tokyo, Japan). THP-1 cells were grown in RPMI-1640 medium supplemented with $10 \%$ foetal bovine serum (Thermo Fisher Scientific, Waltham, MA, USA), 3\% L-glutamine, and 10\% sodium hydrogen carbonate. To induce differentiation into macrophage-like cells, they were incubated with $400 \mathrm{ng} / \mathrm{ml}$ 12-O-tetradecanoyl-13acetate (TPA; Sigma-Aldrich, St. Louis, MO, USA) for $24 \mathrm{~h}$.

Lifeact-GFP expressing THP-1 cells (Lifeact-THP-1). Lifeact is a 17-amino acid actin-binding peptide and a good F-actin reporter (7). THP-1 cells, which stably expressed Lifeact-GFP, were obtained via retroviral infection as described previously (8).

Scanning electron microscopy (SEM) observations. Differentiated macrophages were pre-activated with MAFs $(100 \mathrm{pg} / \mathrm{ml}$ lipopolysaccharides (LPS; Sigma-Aldrich) + $20 \mathrm{ng} / \mathrm{ml}$ human recombinant interferon-gamma (IFN- $\gamma$; Wako, Osaka, Japan) or $8.17 \mu \mathrm{g} / \mathrm{ml}$ serum-MAF) for $4 \mathrm{~h}$. After washing with serum-free RPMI-1640, the medium was changed to serum-free RPMI-1640 containing $3 \mu \mathrm{g} / \mu \mathrm{l}$ magnetic beads (Thermo Fisher Scientific), and incubated for $1 \mathrm{~h}$. Magnetic beads quickly settle on the dish bottom and were used in a novel experimental system for quantitatively evaluating the phagocytic activity of macrophages (4). Cells were fixed with standard methods and SEM observations were conducted 
Table I. Quantitative analyses of SEM images*.

\begin{tabular}{|c|c|c|c|c|c|c|}
\hline \multirow[t]{2}{*}{ MAFs } & \multirow{2}{*}{$\begin{array}{l}\text { Number of } \\
\text { cells }\end{array}$} & \multicolumn{4}{|c|}{ Number of beads } & \multirow{2}{*}{$\begin{array}{l}\text { Engulfing ratio or } \\
\text { cell edge }(\%)\end{array}$} \\
\hline & & $\begin{array}{l}\text { In or on } \\
\text { the cells }\end{array}$ & $\begin{array}{l}\text { On the } \\
\text { edge }^{\dagger}\end{array}$ & $\begin{array}{l}\text { Attached to } \\
\text { the edge }\end{array}$ & $\begin{array}{l}\text { Engulfing } \\
\text { on the edge }\end{array}$ & \\
\hline Without MAFs & 325 & 603 & 167 & 146 & 21 & 12.6 \\
\hline $\mathrm{LPS}+\mathrm{IFN}-\gamma$ & 330 & 734 & 208 & 191 & 17 & 8.1 \\
\hline Serum-MAF & 353 & 842 & 177 & 100 & 77 & 43.6 \\
\hline
\end{tabular}

*Data obtained from 20 SEM images for each MAF; $†$ Number of beads found on the cell edge. This number is the sum of the following two columns; IThis value is calculated from the following formula; (engulfing on the edge) $\times 100 /$ (beads on the edge).

at the Hanaichi UltraStructure Research Institute (Okazaki, Japan) using JSM-6320F (JEOL Ltd., Tokyo, Japan) at $5 \mathrm{kV}$.

Confocal microscopy (CM) observations. Differentiated macrophages were placed in an 8-well slide glass chamber, preactivated, and fed with magnetic beads as described in the previous section. Cells were observed under an LSM700 confocal microscope (Carl Zeiss, Oberkochen, Baden-Württemberg, Germany) and optical sections were obtained at $0.15 \mu \mathrm{m}$ intervals, starting from the bottom of the well and proceeding to the apex of the cell. Timelapse images were obtained at every $30 \mathrm{sec}$ starting from just after addition of serum-MAF at $37^{\circ} \mathrm{C}$.

Quantitative analysis of F-actin accumulation. F-actin accumulation in Lifeact-THP-1 was quantitatively analyzed using imageJ /FIJI (National Institutes of Health, Bethesda, MD, USA). Images were contrast-enhanced (subtract: 60, extract: 2.0) and F-actin accumulation was extracted via an ImageJ ridge-detection plug-in and quantitated as the length of the edge. Actin accumulation was defined as the sum of extracted edge lengths of 20 optical sections within a range of $3 \mu \mathrm{m}$ from the glass bottom.

\section{Results}

Different MAFs induced different morphologies in phagocytosing macrophages. Macrophages, activated via LPS + IFN- $\gamma$ or serum-MAF, were observed under a scanning electron microscope (SEM). Untreated macrophages (control) were small and round, with only a few small extended lamellipodia (Figure 1A). By contrast, LPS + IFN- $\gamma$ or serum-MAF treated cells showed mostly extended lamellipodia from the entire periphery of the cell (Figure 1B and C). Compared with the LPS + IFN- $\gamma$ treated macrophages, serum-MAF treated macrophages displayed much more active membrane ruffling at the sites where beads were engulfed (Figure 1D-F). We designated these intricately ruffed edges of lamellipodia, which consisted of multiple overlapping membranes, as "frill-like structures". Quantitative analysis of the engulfing position indicated that serum-MAF activated macrophages preferentially engulfed beads at the tip of lamellipodia (Table I). Moreover, in serum-MAF activated macrophages, most engulfed beads
$(68.8 \% ; 53$ out of 77$)$ were found within the frill-like structures, suggesting that the frill-like structures were responsible for enhancing the engulfment efficiency.

Frill-like structures were formed by the extensive accumulation of $F$-actin. In general, membrane ruffles and lamellipodia are organized by the actin cytoskeleton. F-actin accumulation was observed in Lifeact-THP-1 macrophages (Figure 2A). While control and LPS + IFN- $\gamma$ activated macrophages exhibited weak fringe-like F-actin accumulation at the cell periphery (Figure 2A, C), serum-MAF activated macrophages showed strong $\mathrm{F}$-actin accumulations in several regions of lamellipodia tips (Figure 2B; white arrowheads). Enlargement of this region (Figure 2D) revealed intricate actin-accumulating edges and multiple stacks of ruffled membrane, suggesting that actin accumulation was associated with frill-like structures. Quantitative analysis of actin accumulation clearly indicated that induction of these characteristic structures was significantly associated with serum-MAF treatment. The length of F-actin accumulations (control; 26.1 $\pm 2.6 \mu \mathrm{m}$, serum-MAF; $38.8 \pm 3.5 \mu \mathrm{m}$, LPS + IFN- $\gamma ; 27.1 \pm 3.1 \mu \mathrm{m}$ : Figure $2 \mathrm{E})$ was significantly different between the control and serum-MAF activated macrophages $(p=0.02)$, as well as between LPS + IFN- $\gamma$ and serum-MAF activated macrophages $(p=0.03$; Student's $t$-test, $\mathrm{n}=3$ ).

$F$-actin accumulation was rapidly induced by serum-MAF. To observe the time course of F-actin accumulation due to serum-MAF, we conducted time-lapse recording of LifeactTHP-1 macrophages, immediately following exposure to serum-MAF. In control macrophages, active movement of the fringe-like actin accumulation occurred; however, their fluorescent intensity did not change (Figure 3A and C). By contrast, expansion of lamellipodia and extrusion of large pseudopods were seen in serum-MAF activated macrophages, even as early as 5 min following serum-MAF addition (Figure 3B). Strong actin accumulation was observed in several regions of the cell periphery at $10 \mathrm{~min}$ following serum-MAF exposure (Figure 3D). Quantitative 

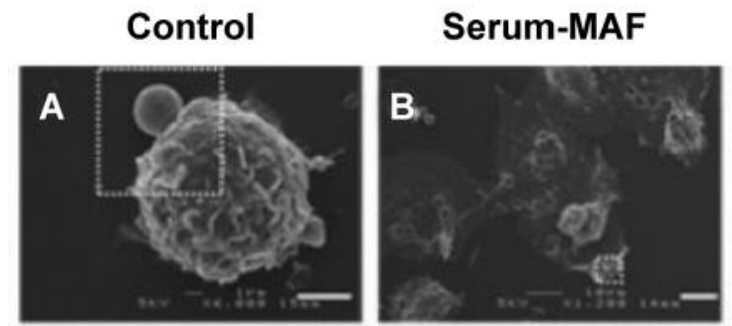

\section{LPS+INF-Y}
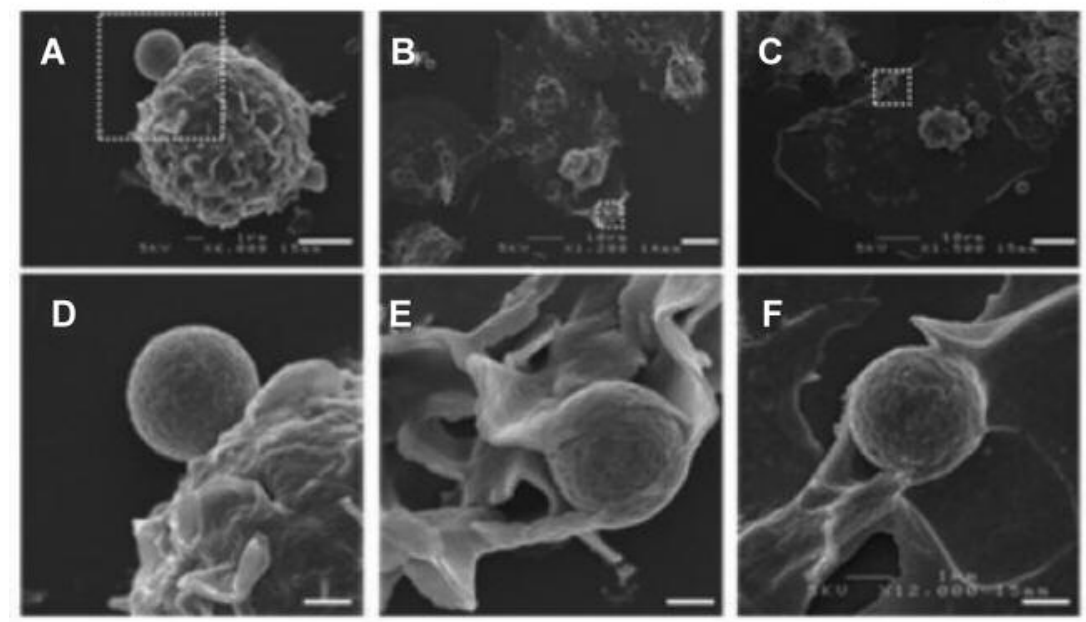

Figure 1. Morphological changes of THP-1-derived macrophages following MAF treatments. After a 4-h treatment with $8.17 \mu \mathrm{g} / \mathrm{ml}$ serum-MAF (B, E), $100 \mathrm{pg} / \mathrm{ml} L P S+20 \mathrm{ng} / \mathrm{ml} I F N-\gamma(C, F)$, or without MAF (A, D), beads were added to macrophages and fixed for SEM observation. Low magnification, whole cell images $(A-C)$ and enlargements of the engulfing region (white rectangle) are shown (D-F). Scale bars; $10 \mu \mathrm{m}$ in $A-C$, $1 \mu \mathrm{m}$ in $D-F$.

Control

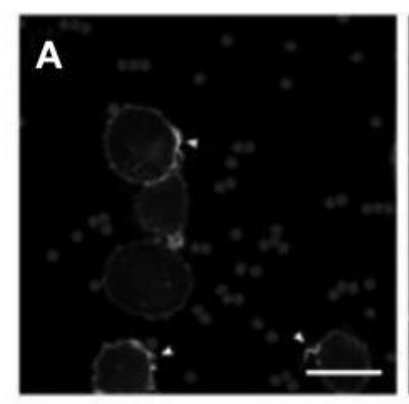

D

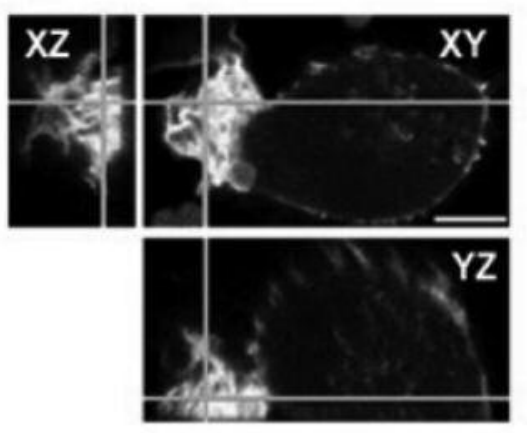

Serum-MAF

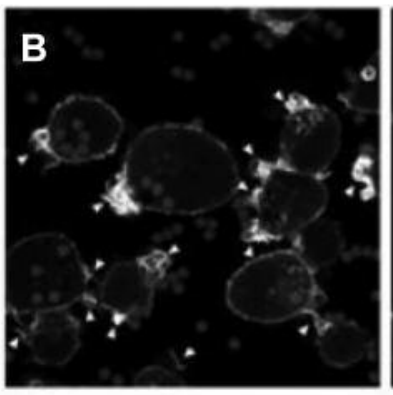

E

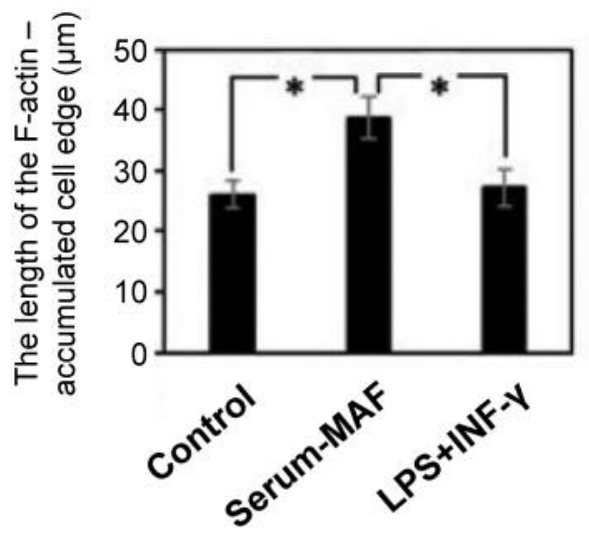

Figure 2. Differences in actin accumulation at the edge of the cells. Confocal images of Lifeact-THP-1 derived macrophages, treated with $8.17 \mu \mathrm{g} / \mathrm{ml} \mathrm{serum-MAF}(B), 100 \mathrm{pg} / \mathrm{ml} L P S+20 \mathrm{ng} / \mathrm{ml} \mathrm{IFN}-\gamma(C)$, or without MAF (A) are shown. White arrowheads indicate actin accumulations in lamellipodia tips. Scale bar; $10 \mu \mathrm{m}$. Three-dimensional analysis of serum-MAF activated macrophage (D) represents intricate membrane ruffling at the site of actin accumulation. Scale bar; $10 \mu \mathrm{m}$. Actin accumulation was quantitatively analyzed using these images $(E)$. Error bars represent SDs. ${ }^{*} p<0.05$ (t-test; $\left.n=3\right)$. 

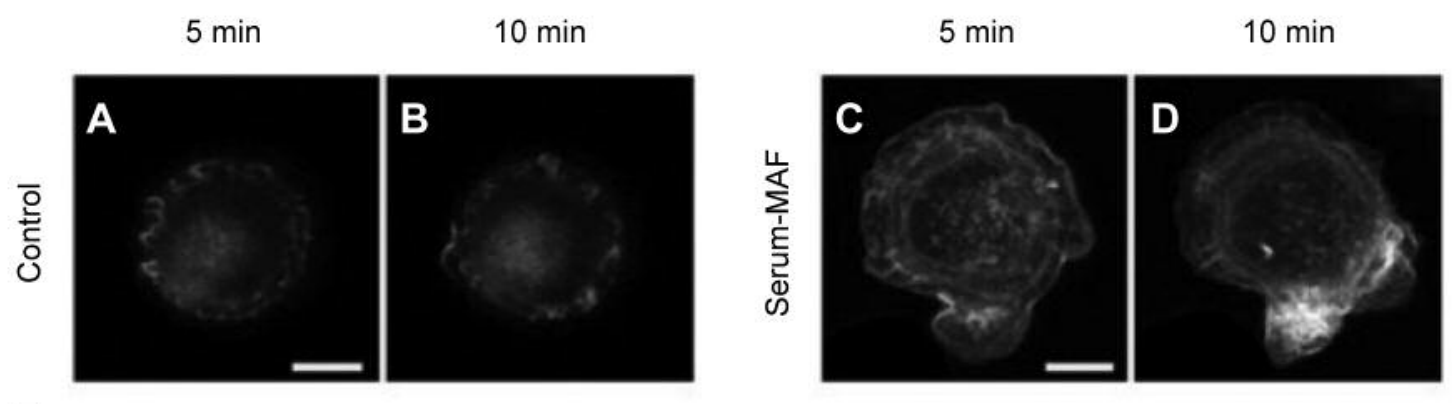

$\mathbf{E}$

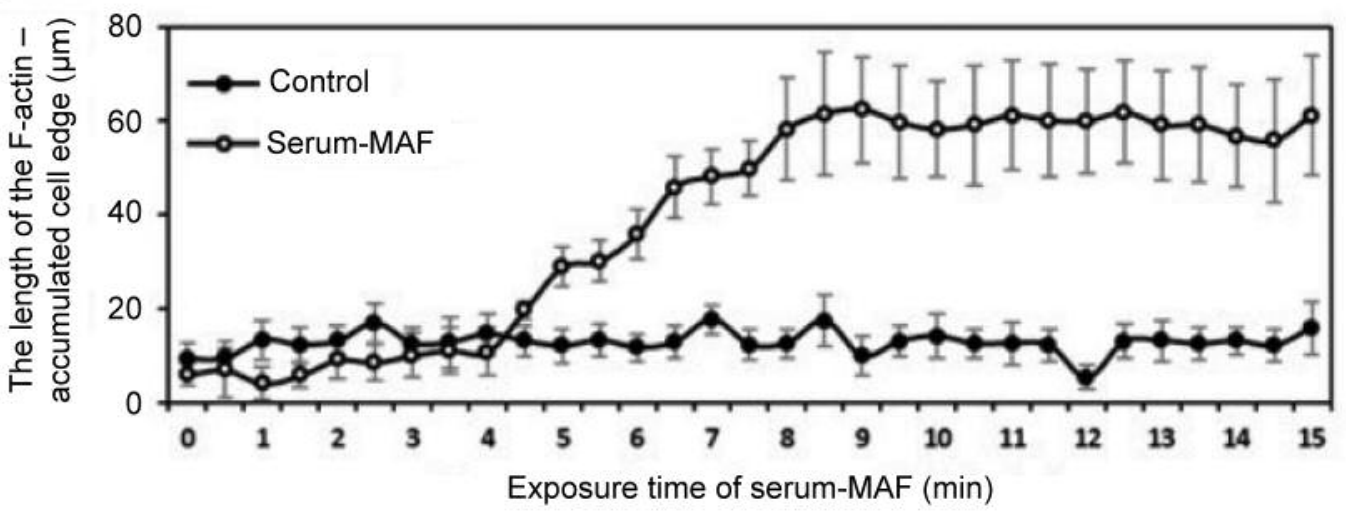

Figure 3. Time lapse of actin accumulation in serum-MAF treated macrophages. Time lapse images of Lifeact-THP-1 derived macrophages 5 min after the start of treatment with serum MAF $(B)$ or without MAF $(A)$ and 10 min post treatment $(D, C)$ are indicated. Scale bar; $10 \mu m .(E)$ Actin accumulation was quantitatively analyzed for each time lapse.

analysis of accumulation at every $30 \mathrm{~s}$ clearly revealed that accumulation occurred very rapidly (Figure 3E). Notably, the fact that no beads were added during this experiment suggested that F-actin accumulation was induced, not by attachment to the beads but by serum-MAF alone.

\section{Discussion}

The current study focused on morphological changes occurring in serum-MAF treated macrophages to determine the processes underlying phagocytic activation. SEM images clearly revealed novel frill-like structures in serum-MAF treated macrophages, while only flat expansion of lamellipodia was observed in LPS + IFN- $\gamma$ treated cells. These findings were consistent with those of a previous report (9). Moreover, the study revealed that serum-MAF activated macrophages preferentially engulfed beads at cell edge, especially using frill-like structures. Therefore, it is conceivable that frill-like structures are involved in the enhancement of engulfment efficiency.

Many studies have addressed the importance of cytoskeletal actin rearrangement in changing the cell shape of macrophages (10). Our confocal images of Lifeact-THP1-derived macrophages revealed strong accumulations of
F-actin only in serum-MAF activated macrophages, where the morphology of actin-accumulated tips of lamellipodia was comparable to that of frill-like structures. Thus, we concluded that vigorous rearrangement of actin cytoskeleton led to the formation of frill-like structures that were observed in serum-MAF activated macrophages. Our time-lapse imaging demonstrated that actin rearrangement in serum-MAF activated macrophages was initiated within 5 min following serum-MAF treatment. This change was very rapid compared with that of the LPS + IFN- $\gamma$ treatment, where 4-24 h elapsed before phenotypical changes became evident (11). To our knowledge, such rapid activation has not been reported previously. Rapid activation within seconds or minutes is very important for innate immunity, in order to counter unpredicted invasion. Findings of this study may further the understanding of activation mechanisms underlying innate immunity, and help promote human health and wellness, with particular reference to the development of novel anticancer immunotherapy.

\section{Conflicts of Interest}

The Authors declare that no conflicts of interest exist. 


\section{Authors' Contributions}

K. K. carried out most of experiments and wrote the initial draft of the manuscript. M. I., R. M., N. K. T., and M. A. contributed to data collection and interpretation. M. I. and T. N. designed the study. T. $\mathrm{N}$. conceived and coordinated of the study and helped to draft the manuscript. All Authors read and approved the final manuscript.

\section{Acknowledgements}

The Authors would like to thank Dr. T. Inui (Inui Immunotherapy Clinic, Osaka, Japan) and Dr. Y. Uto (Tokushima University Graduate School, Tokushima, Japan) for providing the serum MAF.

\section{References}

1 Medzhitov R and Janeway C: Innate immunity. N Engl J Med 343(5): 338-344, 2000. PMID: 10922424. DOI: 10.1056/NEJ M200008033430506

2 Ling $\mathrm{Z}$ and Cheng-Cai W: Inflammatory response of macrophages in infection. Hepatobilliary Pancreat Dis Int 13(2): 138-152, 2014. PMID: 24686541.

3 Emil RU: Antigen-presenting function of the macrophage. Annu Rev Immunol 2: 395-428, 1984. PMID: 6242349. DOI: 10.1146/ annurev.iy.02.040184.00243.

4 Ishikawa M, Mashiba R, Kawakatsu K, Tran NK and Nishikata T: A high-throughput quantitative assay system for macrophage phagocytic activity. Macrophage 5: e1627, 2018. DOI: 10.14800/Macrophage.1627

5 Yamamoto $\mathrm{N}$ and Homma S: Vitamin D3 binding protein (group specific component) is precursor for the macrophage-activating signal factor from lysophosphotidylcholine-treated lymphocytes. Proc Natl Acad Sci USA 88(19): 8539-8543, 1991. PMID: 1924312. DOI: $10.1073 /$ pnas
6 Mashiba R, Ishikawa M, Sumiya Y, Kawakatsu K, Tran NK and Nishikata T: Phagocytic activation of macrophages with serum MAF depends on engulfment efficiency and not migratory activity. Anticancer Res 38(7): 4295-4298, 2018. PMID: 29970564. DOI: 10.21873/anticanres.12727

7 Riedl J, Crevenna AH, Kessenbrock K, Yu JH, Neukirchen D, Bista M, Bradke F, Jenne D, Holak TA, Werb Z, Sixt M and Wedlich-Soldner R: Lifeact: a versatile marker to visualize Factin. Nat Methods 5(7): 605-607, 2008. PMID: 18536722. DOI: $10.1038 /$ nmeth. 1220

8 Kawauchi K, Araki K, Tobiume K and Tanaka N: p53 regulates glucose metabolism through an IKK-NF-kB pathway and inhibits cell transformation. Nat Cell Biol 10(5): 611-618, 2008. PMID: 18391940 . DOI: $10.1038 / \mathrm{ncb} 1724$

9 McWhorter FY, Wang T, Nguyen P, Chung T and Liu WF: Modulation of macrophage phenotype by cell shape. Proc Natl Acad Sci USA 110(43): 17253-17258, 2013. PMID: 24101477. DOI: $10.1073 /$ pnas.1308887110

10 Allen WE, Jones GE, Pollard JW and Ridley AJ: Rho, Rac and Cdc42 regulate actin organization and cell adhesion in macrophages. J Cell Sci 110(6): 707-720, 1997. PMID: 9099945.

11 Mohamad D, Suppian R and Mohd NN: Immunomodulatory effects of recombinant BCG expressing MSP-1C of Plasmodium falciparum on LPS- or LPS+IFN- $\gamma$-stimulated J774A. 1 cells. Hum Vaccin Immunother 10(7): 1880-1886, 2014. PMID: 25424796. DOI: $10.4161 /$ hv.28695
Received May 23, 2019

Revised June 23, 2019

Accepted June 24, 2019 